\title{
Analytical Research of Buoy Motion and Power Response of the Floating-Type Double-Hinged Wave Energy Converter
}

\author{
Biao Li and Hongtao Gao* \\ The College of Marine Engineering, Dalian Maritime University, 116026, Dalian, China
}

\begin{abstract}
The floating-type double-hinged wave energy converter (WEC) is developed to realize the conversion of wave energy. Based on the linear wave theory and wave-body coupled interaction, the nonlinear coupled equations of the heaving-pitching motions for the buoys are established. Under the condition of entirely linear PTO (power take-off system), the analytical solutions of pitching angles are deduced. Then the amplitude-frequency response characteristics of pitching motion and transfer power are discussed. When the collision chocks are installed in the hydraulic PTO, the time-domain changing laws of pitching angles and angular velocity are calculated by using numerical methods. The results show that the coupling effects are significant for the heaving-pitching motion. With the realistic hydraulic PTO, the average transfer power is about $11.5 \%$ lower than that of entirely linear PTO, and pitching motions of buoys show the complex periodic characteristics with wave period.
\end{abstract}

\section{Introduction}

Ocean wave energy is regarded as one of the most promising renewable energy sources for its advantages of substantial deposits and high energy quality, particularly in some oceanic countries such as Ireland, Denmark, Portugal, the United Kingdom, and the United States [1, 2]. In China, wave energy provides an important source of renewable energy in the white paper on China's energy policy (2012). The renewable energies account for the overall target of a 15\% share of total energy by 2020 [3]. A large variety of wave energy converters (WECs) have been proposed to harvest wave energy over the years [4, 5] with a few commercialized and market agreement, such as the raft-type WEC "Pelamis" developed by the Scottish company Pelamis Wave Power (PWP) [6] in UK and the offshore floating type WEC "Mighty whale" [7] designed by Japan marine science and technology centre (JAMSTEC). The maximum power efficiency of "mighty whale" reached $12 \%$.

In this respect, research team of Professor H. T. Gao, Dalian Maritime University in China, has developed a new kind of floating multi-section WEC [8] which is taken as the research object of this paper. Figure 1 is the configuration diagram of this WEC. The device is constituted of multiple section cylindrical buoys that are hinged together by several hydraulic energy conversion units. Wave energy is gathered by buoys in term of resonance phenomenon, Hydraulic cylinder pistons are driven to do reciprocating motion with the change of the angles caused by the non-synchronous six degree of freedom (6-DOF) motions of buoys. Several sea tests with small-sized devices have been carried out in Dalian sea area in 2012 and 2013. The maximum output power achieved $100 \mathrm{~W}$ in the sea tests of 2013 when the buoy length is $4.0 \mathrm{~m}$ and the radius is $0.5 \mathrm{~m}$ in the wave height of about $0.5 \mathrm{~m}$. The results confirmed that the WEC has good practicability and application prospect.

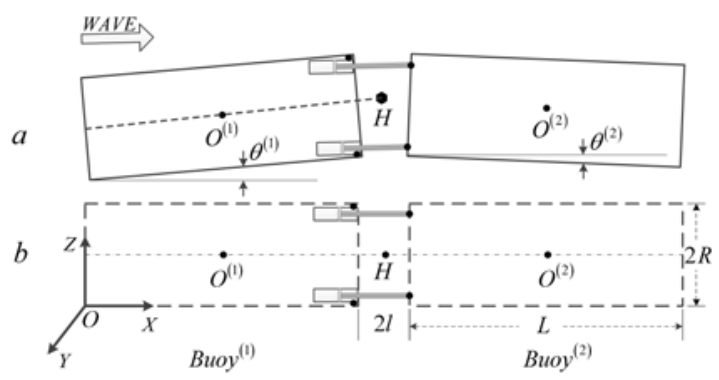

Figure 1. Configuration diagram of the WEC

At present, the research on this type of WEC is concentrated in the numerical simulation and model test. "Pelamis" have been conducted on several sea tests in 2004 [6]. W. C. Chen and Y. L. Zhang presumed that the pitching motions of the rafts are axisymmetric in research of the conversion efficiency of raft-type WEC [9]. F. Hedberg and M. Saccullo discussed the optimal design for a raft-type double-hinged floating WEC by numerical simulations considering linear regular waves with an incident angle of zero $[10,11]$.

In this paper, a valid and practical method for theoretical calculation in the form of differential equations with the help of analytical and numerical technique is presented to construct the motion model of the WEC and discuss the motion characteristics and power responses.

\section{Coupled equations of heaving-pitching motion}

The incidence angle of wave usually approaches 0 under the action of waves, wind and mooring system. So the 6DOF motion of buoys is simplified into 3-DOF motion (heaving, surging and pitching). The surging motion can be ignored for the small motion amplitude and unconspicuous coupling effect. Therefore, the heavingpitching motion is the focus of our research. The key to the research is the wave-body interaction.

As shown in Figure 1, the Buoy ${ }^{(1)}$ and Buoy ${ }^{(2)}$ are equal of length $(L)$ and weight $(m)$ that evenly distributed. 
Based on rigid body kinematics, the motions are represented in a set of equations by the Newton-Euler method.

Heaving motions:

$\left(M^{(n)}+A_{z}^{(n)}\right) \cdot \ddot{z}^{(n)}=\left(G^{(n)}+F_{f}^{(n)}+F_{h}^{(n)}+F_{s}^{(n)}+F_{c}^{(n)}\right) \cdot e_{z}$

Pitching motions:

$\left(I_{y y}{ }^{(n)}+I_{a y y}{ }^{(n)}\right) \cdot \ddot{\theta}^{(n)}=\left[N_{p}+s \times\left(F_{1}+F_{2}\right)\right] \cdot e_{y}$

Where $n=1$ and $n=2$ represent the physical variables of the Buoy ${ }^{(1)}$ and $B u o y^{(2)}$ respectively. $M$ is the mass of buoy, $A_{z}$ is the added mass components, $G$ is the gravity, $F_{h}$ is the hydrodynamic forces. $F_{s}$ is hydrostatic restoring force, $F_{f}$ is buoyancy of buoys, $F_{c}$ is the damping force of power take-off system (PTO). $e_{y}$ and $e_{z}$ are the unit vectors of $\mathrm{Y}$-axis and $\mathrm{Z}$-axis.

$I_{y y}$ and $I_{a y y}$ are the moment of inertia and added moment of inertia to $\mathrm{Y}$-axis. $\ddot{\theta}$ is the pitching angular acceleration of buoys. $F_{1}$ and $F_{2}$ are the forces of the hydraulic cylinder piston rods. $s$ is the vector distance between rotation axis and geometric center of piston rod. $N_{p}$ represent the total moment of the fluid pressure and the gravity on the center of gravity of buoy. The following expression can be denoted by force analysis.

$N_{p}=\int_{-L-l}^{-l}\left(f_{h z}{ }^{(n)}+f_{s}^{(n)}+f_{f}^{(n)}\right) d x \cdot \cos \theta^{(n)}+$

$\int_{0}^{|s|} f_{h x}^{(n)} d z+G^{(n)} \cdot(l+L / 2)$

Where $f_{h z}{ }^{(n)}$ and $f_{h x}{ }^{(n)}$ represent the infinitesimals of the vertical and horizontal components of the hydrodynamic force $F_{f} \cdot f_{f}^{(n)}$ and $f_{s}^{(n)}$ represent the infinitesimals of the buoyancy $F_{f}$ and hydrostatic restoring force $F_{s}$.

The hydrostatic restoring force is denoted as:

$F_{s}=-\iint \rho g \eta d s=-\rho g S_{w p} \cdot z[12]$

Here $S_{w p}$ is the mean wetted surface area of buoys in still water.

The vertical component of PTO force is:

$F_{c}^{(n)} \cdot e_{z}=\left(F_{1}-F_{2}\right) \sin \frac{\theta^{(1)}+\theta^{(2)}}{2}$

The formulas (1), (2) are the coupled equations of heaving-pitching motion.

\section{Calculation results and analysis}

The PTO is modeled both by a simplified system of an entirely linear damper and also by a more realistic system of a hydraulic piston with a certain retractable length. In the simplified linear model of the PTO, the coupled equations of heaving-pitching motion are expected to be solved using analytical solution method. Then the motion characteristics and power responses are analyzed. For a realistic system, numerical solution method would almost the best way to conduct its time-domain analysis and power distribution.

To simplify the calculating process, the linear potential theory is selected and the wave attenuation is neglected.

The main parameters of WEC and sea condition are shown in Table 1 and Table 2.
Table 1. Main parameters of WEC

\begin{tabular}{|l|l|}
\hline Structure Parameter & Value \\
\hline Shape of buoy & cylinder \\
\hline Length of buoy $/ L$ & $8.0 \mathrm{~m}$ \\
\hline Radius of buoy/ $B$ & $1.0 \mathrm{~m}$ \\
\hline Draft $\mathrm{t}$ of buoy $/ d$ & $1.0 \mathrm{~m}$ \\
\hline Damping coefficient $/ C$ & $50000 \mathrm{Ns} / \mathrm{m}$ \\
\hline
\end{tabular}

Table 2. Conditions of China Yellow Sea and Bohai Sea [13]

\begin{tabular}{|l|l|}
\hline Parameter & Value \\
\hline Wave type & linear wave \\
\hline Wave direction & $0^{\circ}$ \\
\hline Wave height $/ H$ & $1.2 \mathrm{~m}$ \\
\hline Wave period $/ T$ & $2.5 \mathrm{~s}-5.0 \mathrm{~s}$ \\
\hline Wave circular frequency $/ \omega$ & $1.26 \mathrm{rad} / \mathrm{s}-2.51 \mathrm{rad} / \mathrm{s}$ \\
\hline Depth $/ d$ & $40 \mathrm{~m}$ \\
\hline Sea-water density $/ \rho$ & $1025 \mathrm{~kg} / \mathrm{m}^{3}$ \\
\hline
\end{tabular}

\subsection{Entirely linear PTO}

According to the decomposition of forces and moments, the pitching motions of buoys is deduced as:

$$
\left\{\begin{aligned}
\left(I_{y y}{ }^{(1)}+I_{a y y}{ }^{(1)}\right) \cdot \ddot{\theta}^{(1)} & =\left(F_{h z}^{(1)}+F_{s}^{(1)}\right) \cdot(L / 2+l) \cos \theta^{(1)}+ \\
& F_{h x}^{(1)} \cdot s-\left(F_{1}+F_{2}\right) \cdot R \cos \theta^{(1)} \\
\left(I_{y y}{ }^{(2)}+I_{a y y}{ }^{(2)}\right) \cdot \ddot{\theta}^{(2)} & =\left(F_{h z}{ }^{(2)}+F_{s}^{(2)}\right) \cdot(L / 2+l) \cos \theta^{(2)}+ \\
& F_{h x}{ }^{(2)} \cdot s+\left(F_{1}+F_{2}\right) \cdot R \cos \theta^{(2)}
\end{aligned}\right.
$$

It is inferred that the pitching motions of buoys are considered as simple harmonic motions. The angular displacements are denoted in complex number:

$\theta^{(n)}=\operatorname{Re}\left[\theta_{m}{ }^{(n)} \exp (-i \omega t)\right]$

Where $\theta_{m}{ }^{(n)}$ is the amplitudes of pitching angle in the form of complex number. $\mathrm{Re}[]$ indicates the real part of complex number.

The hydrodynamic force is described as follow [14].

$F_{h}=\operatorname{Re}\left[F_{0} \exp (-i \omega t)\right]$

In which $F_{0}$ is the amplitude of hydrodynamic force that is unrelated to time term.

Plug formula (7) and (8) into equations (6), then eliminate time term. The analytic solutions of pitching angle amplitudes are obtained:

$$
\begin{aligned}
& \theta_{m}^{(1)}=-\frac{\left(F_{0}^{(1)}-\rho g S_{w p} \cdot z_{0}^{(1)}\right) \cdot(L / 2+l)-2 C R^{2} \theta_{m}^{(2)} \cdot i \omega}{2 C R^{2} \cdot i \omega+\left(I_{y y}{ }^{(1)}+I_{a y y}{ }^{(1)}\right) \cdot \omega^{2}} \\
& \theta_{m}^{(2)}=-\frac{A \cdot(L / 2+l)}{\left(I_{y y}{ }^{(1)}+I_{a y y}{ }^{(1)}\right)^{2} \cdot \omega^{3}+4 C R^{2} \cdot i \cdot \omega^{2} \cdot\left(I_{y y}{ }^{(1)}+I_{a y y}{ }^{(1)}\right)}
\end{aligned}
$$

$A=\left(\exp (i \omega \Delta t) \cdot F_{0}^{(1)}-\rho g S_{w p} \cdot z_{0}^{(2)}\right)$

$\left(2 C R^{2} \cdot i+\left(I_{y y}{ }^{(1)}+I_{a y y}{ }^{(1)}\right) \cdot \omega\right)+2 C R^{2} \cdot i \cdot\left(F_{0}^{(1)}-\rho g S_{w p} \cdot z_{0}^{(1)}\right)$

Here $z_{0}{ }^{(1)}$ and $z_{0}{ }^{(2)}$ are the displacement amplitudes of heaving motions of $B u o y^{(I)}$ and Buoy ${ }^{(2)}$.

The wave energy transfer power of PTO is expressed by the following formula.

$\bar{P}_{C} \approx \int_{0}^{T} 2 C \cdot v_{C}^{2} d t \approx \int_{0}^{T} 2 C \cdot\left(R \cdot \theta^{(r e l)}\right)^{2} d t=C\left(R \theta_{m}^{(r e l)} \omega\right)^{2}$ 
Where $\bar{P}_{C}$ is average wave energy transfer power, $\theta_{m}^{(r e l)}$ is the amplitude of relative pitching angle $\left(\theta^{(r e l)}\right)$, $\theta^{(r e l)}=\theta^{(1)}-\theta^{(2)}$.

Figure 2 shows the amplitude-frequency responses of pitching motion and transfer power. The amplitude of pitching angle attains the peak at the frequency of about $2.3 \mathrm{rad} / \mathrm{s}$, and then decline rapidly. The reason for this is that, there are strong coupling effects between the pitching motion and heaving motion. And the main factor which affects the heaving motion response is the proximity of the natural frequency of buoys to the wave period. The resonance can be thought to occur at this frequency.

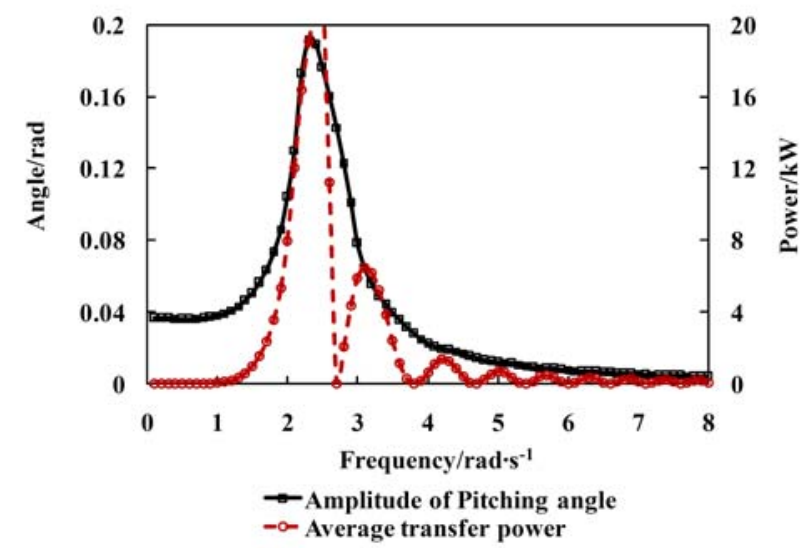

Figure 2. Amplitude-frequency responses of pitching motion and transfer power

For the average transfer power, the peak power is discovered at the frequency of $2.6 \mathrm{rad} / \mathrm{s}$. When the frequency exceeds $2.6 \mathrm{rad} / \mathrm{s}$, the power overall showes a downward trend quickly but with multiple fluctuations.

\subsection{Hydraulic PTO}

As stated before, for a realistic system of a hydraulic piston, collision chocks are usually installed to prevent damages of buoys and PTOs in bad sea condition. Under this condition, the maximum relative pitching angle is fixed that the hydraulic pistons are limited in a certain length. The collision process of buoys satisfies the angular momentum theorem.

$$
\begin{aligned}
& \left(I_{y y}{ }^{(1)}+I_{a y y}{ }^{(1)}\right) \cdot \theta^{(1)}(t+\Delta t)+\left(I_{y y}{ }^{(2)}+I_{a y y}{ }^{(2)}\right) \cdot \theta^{(2)}(t+\Delta t)= \\
& \left(I_{y y}{ }^{(1)}+I_{a y y}{ }^{(1)}\right) \cdot \theta^{(1)}(t)+\left(I_{y y}{ }^{(2)}+I_{a y y}{ }^{(2)}\right) \cdot \theta^{(2)}(t)+\Delta L(\Delta t)
\end{aligned}
$$

Where $\Delta t$ is time step, $\Delta L(\Delta t)$ is the increments of primary moment in $\Delta t$ time.

Figure 3 shows the time-domain curves of pitching motion with hydraulic PTO. The pitching motions of buoys exhibit the complex periodic characteristics with evident sloping platforms at the larger angles. This characteristic is the reason that the contact impact occurs when the relative pitching angle exceeds the threshold. Their periodicities are still of the same with wave period. Figure 4 is the curve of corresponding angular velocity.

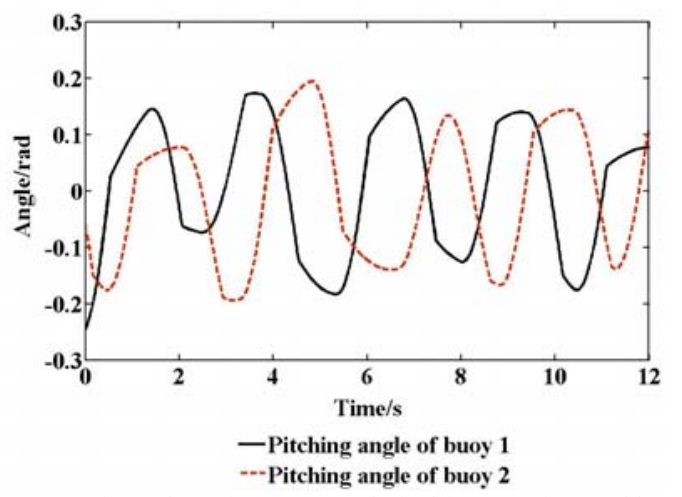

Figure 3. Time-domain curves of pitching motions of buoys with hydraulic PTO

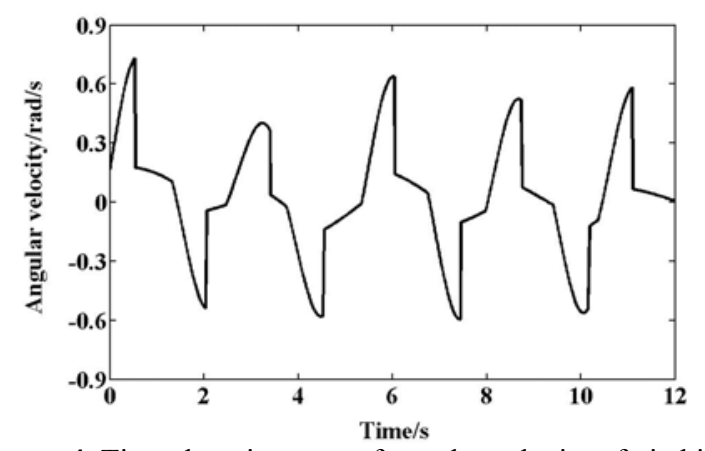

Figure 4. Time-domain curve of angular velocity of pitching motion with hydraulic PTO

\subsection{Transfer power analysis}

Figure 5 and figure 6 show the changes of transfer power with entirely linear PTO and hydraulic PTO. For the two type of PTOs, both the curves take on periodic behaviors with half of wave period. But with entirely linear PTO, the change of curve shows more orderly than the curve with hydraulic PTO. And there are platforms at the larger power. The numerical results show that the average transfer power is $20.8 \mathrm{~kW}$ with entirely linear PTO and the power is $18.4 \mathrm{~kW}$ with hydraulic PTO. The loss rate of power is about $11.5 \%$.

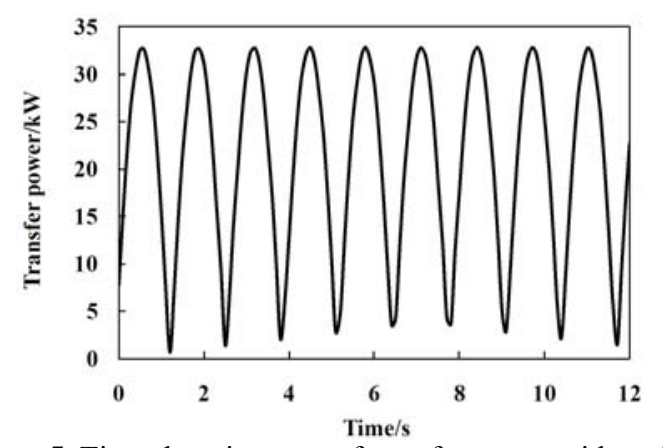

Figure 5. Time-domain curve of transfer power with entirely linear PTO 


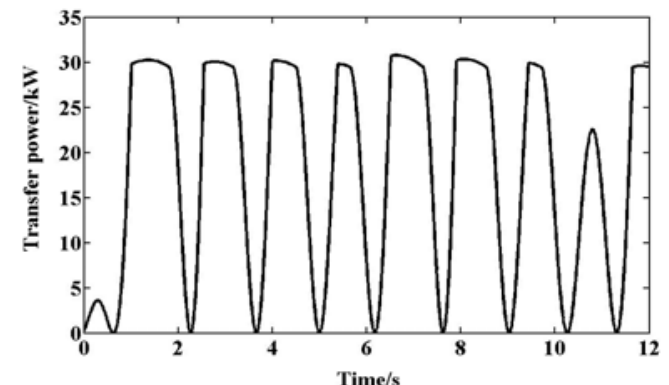

Figure 6. Time-domain curve of transfer power with hydraulic PTO

\section{Conclusions}

This paper takes the floating-type double-hinged WEC as research object. The coupled equations of heavingpitching motion of wave capture buoys are established to analyze the motion characteristics and power responses The main conclusions are as follows:

(1) The coupling effects between the pitching motion and heaving motion are strong. The natural frequency of buoys is a main factor that affects the coupling motions and power responses.

(2) For a realistic system of a hydraulic piston installed collision chocks, the pitching motions of buoys exhibit the complex periodic characteristics with wave period. There are always evident sloping platforms in the curves.

(3) The transfer power with the realistic hydraulic PTO will decrease compared with the entirely linear PTO. The loss rate of power is about $11.5 \%$ for this WEC.

\section{Acknowledgements}

This work was financially supported by the Research Funds of the Maritime Safety Administration of the People's Republic of China (2012 26) and the Fundamental Research Funds for the Central Universities (3132014338).

\section{References}

[1] B. Godfrey. Renewable Energy. Oxford: Oxford University Press. 298-337 (2004)

[2] Y. Gong. Development trend of wave power generation technology in the world. POWER DSM. 10: 71-72(2008)

[3] State Council of the People's Republic of China. Beijing: China's energy policy (2012)

[4] T. Heath, T. Whittake, C. Boake. The design, construction and operation of the LIMPET wave energy converter. In: Proceedings of the 4th European wave energy conference. 49-55 (2000)

[5] A. Clément, P. McCullen, A. Falcão, A. Fiorentino, F. Gardner, K. Hammarlund, et al. Wave energy in Europe: current status and perspectives. RENEW SUST ENERG REV, 6: 405-31 (2002)

[6] R. Yemm, D. Pizer, C. Retzler, etc. Pelamis: experience from concept to connection. PHILOS T R
SOC A. 370: 365-380 (2012)

[7] I. Masaaaki, W. Yukihisa, O. Hirotaka. Development of an offshore floating type wave power energy converter system "mighty whale". SCI TECH JPN. 60: 28-30 (1997)

[8] H. T. Gao, B. Li. ESTABLISHMENT OF MOTION MODEL FOR WAVE CAPTURE BUOY AND RESEARCH ON HYDRODYNAMIC PERFORMANCE OF FLOATING-TYPE WAVE ENERGY CONVERTER. POL MARIT RES. 22: 106-111 (2015)

[9] W. C. Chen, Y. L. Zhang. Numerical study on conversion efficiency from wave energy to hydraulic energy by raft-type wave energy convertors. J Hydroelec Eng. 32: 191-196 (2013)

[10] F. Hedberg, M. Saccullo. Design of a numerical model to analyse a wave energy dissipation device for hydropower. Master's thesis. Chalmers University of Technology (2014)

[11] H. J. Koh, W. S. Ruy, I. H. Cho, etc. Multi-objective optimum design of a buoy for the resonant-type wave energy converter. J Mar Sci Technol. 20: 53-63 (2015)

[12] M. E. Mccormick. A modified linear Analysis of a Wave-Energy Conversion Buoy. OCEAN ENG. 3: 133-144 (1976)

[13] L. J. Sun, C. Gao. Statistics and analysis on storm and climate of Yellow Sea and the Bohai Sea. World Shipping. 27: 18-19 (2004) 